\title{
Investigation of the Effect of Number of Longitudinal Rounded Grooves on Hydrodynamic Forces acting on Cylinders in Cross Flow at $\mathrm{Re}=10^{5}$
}

\author{
Oktay YILMAZ \\ Ylldız Technical University, Faculty of Naval Architecture and Maritime, Turkey \\ oktay@yildiz.edu.tr; ORCID ID: https://orcid.org/0000-0002-3260-2992
}

\begin{abstract}
Crossflow over cylindrical structures has been extensively studied by both academia and industry in various fields. It is responsible for in-line and transverse vibrations of marine risers. Flow-induced structural vibrations may end up with the failure of these tubular systems in oceans and seas. First experimental studies in literature signified that longitudinally grooved cylinders inspired by Saguaro trees may offer reductions both in drag and lift forces. In the present study, the performance of 2 dimensional (2D) unsteady Reynolds averaged Navier-Stokes (URANS) simulations is tested in predicting hydrodynamic force coefficients of cylinders. First, CFD runs are conducted on a smooth cylinder and a cactus-like cylinder in literature. Then flow over 8-, 10-, and 12-grooved cylinders with a diameter of $0,5 \mathrm{~m}$ are solved to analyze flow features in detail and evaluate the capability of 2D URANS solver for the problem at hand. $\mathrm{k}-\omega$ SST turbulence model is employed in URANS solver. It is shown that as the number of grooves increases, mean drag slightly decreases and amplitude of drag decreases by $29 \%$. Separation angle is significantly improved with a number of grooves from $92^{\circ}$ to $120^{\circ}$. However, the amplitude of lift force increases with the number of grooves with reference to 2D CFD simulations.
\end{abstract}

\section{Keywords}

Cylinder, Longitudinal Rounded Grooves, Flow Induced Forces, CFD, Marine Riser.

\section{Introduction}

Cylindrical-shaped bodies in crossflow stream are subject to unsteady aerodynamic fluctuating drag and lift forces due to arising vortex shedding behind the object [1]. In response, the cylindrical structure fluctuates in streamwise and transverse directions [2],[3]. The cylindrical body may be an antenna, a riser in the ocean or supporting column of a floating petroleum platform in the sea. When vortex shedding frequency coincides with the natural frequency of the body, amplitudes of motion grow substantially and may result in the

To cite this article: Yilmaz, O. (2020). Investigation of the Effect of Number of Longitudinal Rounded Grooves on Hydrodynamic Forces acting on Cylinders in Cross Flow at $\mathrm{Re}=10^{5}$. Journal of ETA Maritime Science, 8(3), 178-193. 
destruction of the structural system. Some engineering solutions have been devised to reduce fluctuating forces such as helical strakes [3] and fairings [4] on marine risers [5]. The main idea behind is to break down synchronous vortex shedding along the cylinder span with helical strakes [2] and to increase backpressure streamlining the body with fairings in order to decrease drag. In the tandem arrangement of two helical straked-cylinders, increase in fluctuating lift on a downstream cylinder was experimentally demonstrated [6]. On the other hand, helical strakes increase drag [7]. Fairings on marine risers reduce adverse pressure gradient on the downstream side, block vortices interactions, limit transverse motion of vortices and move away vortices from the body. These features diminish both mean drag and fluctuating lift forces [8]. Risers fitted with fairings are not suitable for use near sea surface due to frequent directional changes of surface waves. These techniques are frequently applied in production and drilling risers in the offshore oil and gas industry to suppress flow-induced vibrations [8]. While helical strakes are omnidirectional, fairings are unidirectional solutions.

There exist some other suggested modifications on the cylinder in scientific research studies. Sinusoidal wavy cylinders [9], circular rings around cylinder [10], cylinders with bumps [7], surface roughened cylinders [11] are in this list. It was revealed that wavy cylinders disrupts regular vortex shedding and increases three-dimensionality in the wake. These impacts yielded considerable decrease both in drag and fluctuating lift [12]. Tripwire or other attachments on cylinders were studied to make boundary layer turbulent before separation and delay flow detachment from the surface [13][14]. However, tripwire techniques are unidirectional; they immediately lose their benefits when the current direction changes. Longitudinal U-grooved cylinders were also experimented and found successful for a reduction in mean drag at Reynolds (Re) numbers greater than $4 \times 10^{4}$. But the Strouhal (St) number slightly increased with Re number [15]. In experiments, longitudinal V-grooves on cylinders decreased the critical Re number where drag coefficient $\left(C_{d}\right)$ drops [16].

Due to the special features of Saguaro trees, they have attracted much attention in recent years [17]-[19]. Saguaro trees have triangular grooves in spanwise direction and spines at the tip [17]. Although roots of Saguaros are very shallow, with an average root-length of $25 \mathrm{~cm}$, these trees can withstand strong winds for years. Their long life ends up to 150-years [17]. Many experimental and computational research studies have been conducted on unsteady hydrodynamic characteristics on Saguaro trees [19]-[23]. It was demonstrated experimentally that both mean drag and root-mean-square (RMS) of lift force on cylinders can be reduced with longitudinal triangular grooves [23]. Grooves as vortex generators ensure attached flow and late separation resulting in less drag [18]. It was also reported that turbulence intensity (TI) around the cacti, which is partially responsible for the fluctuating forces on bluff bodies, decreased [18][19][22].

To the author's knowledge, aero/ hydrodynamic characteristics of the longitudinal rounded grooved cylinder have not been investigated numerically before. Though fluctuating lift has been more widely researched, fluctuating drag forces damage the structure in the same level with their double frequency [24]. In this study, the effects of the number of rounded grooves on fluctuating lift and drag forces are investigated by 2D URANS Computational Fluid Dynamics (CFD) analyses. CFD simulations are first performed for flow around smooth and 8-grooved cylinders to test CFD solver in predicting flow field 
and aero/hydrodynamic characteristics comparing with literature experimental data.

\section{CFD Simulations}

In this study, CFD simulations are carried out for one smooth cylinder and three grooved cylinders. 2D computational domain and its boundaries are shown in Figure 1a. Free stream velocity $(U)$ and TI $(\% 0.35)$ are specified at the inlet. Since derivatives of variables in a normal direction to the farfield boundary are expected to vanish, the symmetry boundary condition is applied. Gage pressure is set to zero at the outlet. No-slip boundary condition is applied on cylinder walls.

CFD simulations are performed by use of Ansys Fluent software [25]. k- $\omega$ SST, a fully turbulent flow model, is employed to model unsteady Reynolds averaged NavierStokes equations. This model is known to be successful in predicting adverse pressure gradient boundary layer flows in which flow separation occurs [26][27]. For a few decades, the k- $\omega$ SST model has been applied to solve turbomachinery flow problems by securing trade-off between computational accuracy and computation time [28]. Continuity and momentum equations derived by RANS modeling are as follows [27],

$$
\begin{aligned}
& \frac{\partial \rho}{\partial t}+\frac{\partial}{\partial x_{i}}\left(\rho u_{i}\right)=0 \\
& \frac{\partial}{\partial t}\left(\rho u_{i}\right)+\frac{\partial}{\partial x_{j}}\left(\rho u_{i} u_{j}\right)= \\
& -\frac{\partial p}{\partial x_{i}}+\frac{\partial}{\partial x_{j}}\left[\mu\left(\frac{\partial u_{i}}{\partial x_{j}}+\frac{\partial u_{j}}{\partial x_{i}}-\frac{2}{3} \delta_{i j} \frac{\partial u_{l}}{\partial x_{i}}\right)\right]+\frac{\partial}{\partial x_{j}}\left(-\rho u_{i} u_{j}\right)
\end{aligned}
$$

Here $\rho, u$, and $p$ are density, time-averaged velocity component, and time-averaged pressure, respectively. The Boussinesq hypothesis is employed to relate the Reynolds stresses with the time-averaged velocity gradients.

$$
-\rho \overline{u_{i}^{\prime} u_{j}^{\prime}}=\mu_{t}\left(\frac{\partial u_{i}}{\partial x_{j}}+\frac{\partial u_{j}}{\partial x_{i}}\right)-\frac{2}{3}\left(\rho k+\mu_{t} \frac{\partial u_{k}}{\partial x_{k}}\right) \delta_{i j}
$$

Here $\mu t(=\rho k / \omega)$ and $k$ are turbulent viscosity and turbulence kinetic energy (TKE), respectively. $\omega$ is the ratio of turbulence dissipation rate $(\varepsilon)$ to $k$. In the Boussinesq hypothesis, $\mu \mathrm{t}$ is assumed to be an isotropic scalar quantity which works well for shear dominated flows. $k$ and $\omega$ equatib3)s are given below [27].

$$
\begin{aligned}
& \frac{\partial}{\partial t}(\rho k)+\frac{\partial}{\partial x_{i}}\left(\rho k u_{i}\right)=\frac{\partial}{\partial x_{i}}\left(\Gamma_{k} \frac{\partial k}{\partial x_{i}}\right)+G_{k}-Y_{k} \\
& \frac{\partial}{\partial t}(\rho \omega)+\frac{\partial}{\partial x_{i}}\left(\rho \omega u_{i}\right)=\frac{\partial}{\partial x_{i}}\left(\Gamma_{\theta} \frac{\partial \omega}{\partial x_{i}}\right)+G_{\omega}-Y_{\omega}+D_{\omega}
\end{aligned}
$$

Here $\Gamma_{k}$ and $\Gamma_{\omega}$ are effective diffusivities of $k$ and $\omega . G_{k}$ and $G_{\omega}$ are the production terms for $k$ and $\omega . Y_{k}$ and $Y_{\omega}$ are the dissipation terms for $k$ and $\omega . D_{\omega}$ is a cross-diffusion term. Further information about the k- $\omega$ SST turbulence model and its model constants can be found in [26][27].

Pressure-based solver with the pressurevelocity coupling algorithm is employed to achieve a converged solution in less number of iteration compared to the segregated algorithm. In spatial discretization, diffusion terms are discretized with a second-order cell-centered scheme and convective terms with a second order upwind scheme.

First-order implicit time integration is applied to advance in the solution of the unsteady problem. Dimensionless time step size $\Delta t^{+}(=\Delta t U / D)$ is 0.011 which is approximately $1 / 200$ of one period of the highest frequency wave of fluctuating forces. D is cylinder diameter. Gradients of scalar and vector variables in CFD computations are calculated employing the least square cellbased method.

Fully structured computational mesh with quad cells is generated by Ansys Meshing 
(Figure 1b). Mesh independence study is conducted for the flow of air around the 8-grooved cylinder at a Reynold number of $1.1 \times 10^{5}$. The coefficients of fluctuating drag and lift forces for varying number of computational cells are provided in Table 1. Relative change between variables of the last two consecutive mesh structures is maximum of $4.6 \%$. To work with reasonable computation time and ensure sufficient grid convergence, the finest mesh structure is employed in CFD computations and no further mesh refinement is carried out. Average mesh skewness and its standard deviation are 0.27 and 0.17 , respectively. Average and maximum aspect ratios of cells are 2.6 and 26, respectively. While average $\mathrm{y}^{+}$value is nearly 1 , maximum $\mathrm{y}^{+}$is smaller than 4 on the cylinder wall. When determining parameters in computations, an iteration convergence study is conducted varying iteration number from 3 to 60 for the time steps. It is seen that 5 number of

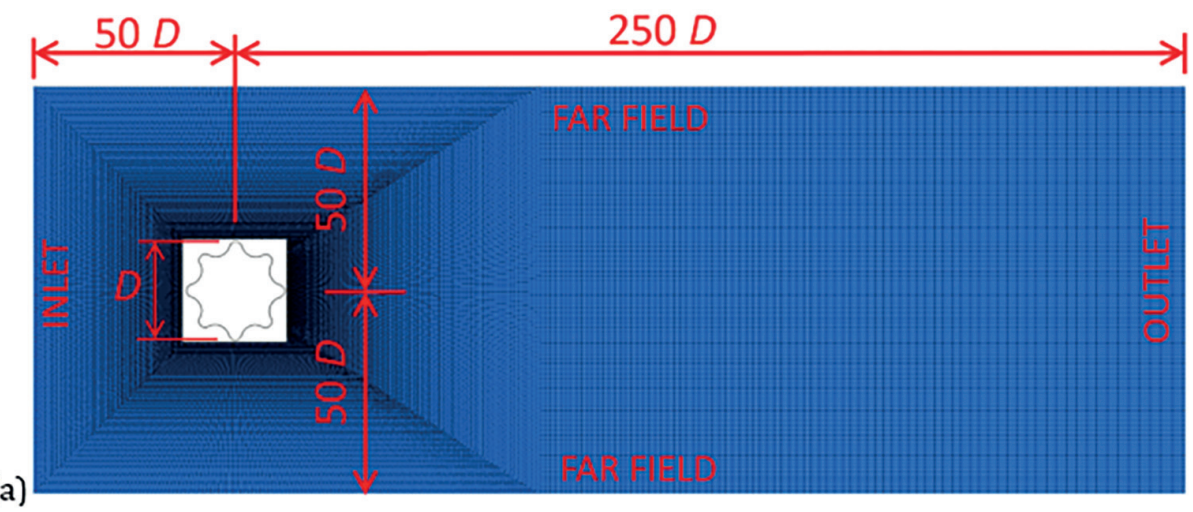

(b)

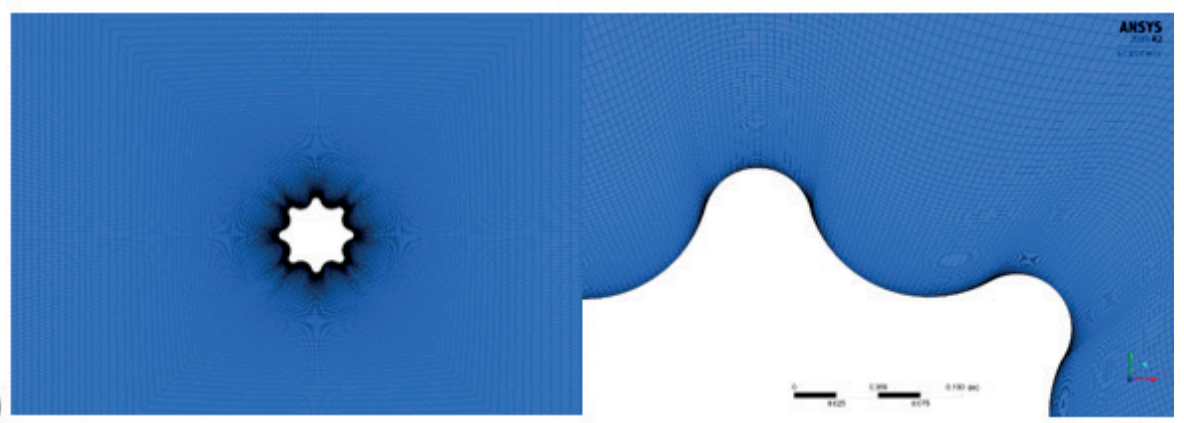

\begin{tabular}{|c|c|c|c|c|c|c|}
\hline Mesh & $\begin{array}{c}\text { Number } \\
\text { of cells }\end{array}$ & $\begin{array}{c}\mathrm{Cd}- \\
\text { mean }\end{array}$ & $\begin{array}{c}\mathrm{Cd}- \\
\mathrm{rms}\end{array}$ & $\begin{array}{c}\mathrm{fd} \\
\mathrm{(Hz})\end{array}$ & $\mathrm{Cl}-\mathrm{rms}$ & $\begin{array}{c}\mathrm{fl} \\
(\mathrm{Hz})\end{array}$ \\
\hline coarse & 63,600 & 1.53 & 0.123 & 173 & 0.876 & 88 \\
\hline medium & 113,880 & 1.52 & 0.131 & 172 & 0.843 & 87 \\
\hline fine & 167,520 & 1.50 & 0.149 & 168 & 0.847 & 84 \\
\hline finest & 253,500 & 1.46 & 0.156 & 164 & 0.841 & 82 \\
\hline
\end{tabular}

iterations at each time step is sufficient to reach a converged solution for the relevant time step. In the current study, 8 number of iterations is implemented for the time steps. The same mesh structure and solver parameters are applied to all cases.

\section{Results and Discussion}

\subsection{Cross Flow over Smooth Cylinder at Re} $=10^{5}$

Although a cylinder has a simple shape, flow characteristics around are very sophisticated and depend on Re number. Flow behaviour around a smooth cylinder is classified as follows [1],[29]-[31].
Table 1. Mesh Independence Study 
- When $\mathrm{Re}<190$ flow is completely laminar both in the wake and boundary layer and, $2 \mathrm{D}$ vortex shedding is observed after $\mathrm{Re}=$ 40.

- Subcritical regime $\left(300<\operatorname{Re}<2 \times 10^{5}\right)$ : Flow is laminar in the boundary layer, turbulent in the wake. Laminar boundary layer separation takes place and regular alternating vortices shed.

- Lower transition $\left(2 \times 10^{5}<\operatorname{Re}<3.5 \times 10^{5}\right)$ : Laminar boundary layer separates and then turbulent shear layer reattaches. The boundary layer separates once again. Laminar separation bubble (LSB) forms on one side of the cylinder wall. The cylinder is subject to large mean lift forces.

- Supercritical regime $\left(3.5 \times 10^{5}<\mathrm{Re}<\right.$ 1.5 $\times 10^{6}$ ): Laminar boundary layer separates and then turbulent shear layer reattaches. Laminar separation bubble forms on the two side of the cylinder wall. St number is between 0.4 and 0.45 [32]. Mean drag is minimum.

- Upper transition $\left(1.5 \times 10^{6}<\mathrm{Re}<4 \times 10^{6}\right)$ : Laminar-turbulent transition occurs within the boundary layer without LSB.

- Transcritical regime $\left(\operatorname{Re}>4 \times 10^{6}\right)$ : Flow is turbulent both in the wake and boundary layer.

Besides the turbulent flow is of threedimensional (3D) character, the flow field around a cylinder has its own special 3D features such as cellular shedding, oblique shedding, vortex dislocations and spanwise momentum transfer by streamwise vortices [1]. Laminar-turbulent transition, LSB, flow separation and 3D wake field make it difficult to predict flow structures around a cylinder by 2D CFD analyses. Performing 3D direct numerical simulations (DNS) is the ideal solution to tackle with the problem, but it requires supercomputers and substantial computation time [33]. 3D CFD simulations of Large Eddy Simulation (LES) or Detached Eddy Simulation turbulence modelling are the alternative solutions. Their computation cost is less than DNS, but they are still computationally costly and very fine mesh structures should be constructed [5]. Therefore, to estimate vortex-induced vibrations of cylindrical structures, experimental tests are more frequently carried out [4][7][22][31][34].

Flow regime around the cylinder at $\mathrm{Re}=$ $10^{5}$ stays in a subcritical region where the boundary layer is laminar. Though k- $\omega$ SST is a turbulence model which considers the flow to be fully turbulent, low Re number terms in it offer very simple laminar-turbulent transition modeling capability [27]. The flow of water over a smooth cylinder at $\mathrm{Re}=10^{5}$ is solved by 2D URANS computations and compared with published literature data in this section. $U=0.275 \mathrm{~m} / \mathrm{s}$ and $\mathrm{D}=0.5 \mathrm{~m}$. Parameters of fluctuating drag and lift forces are listed in Table 2. Strouhal number, $\mathrm{St}=\mathrm{f}_{1}$ D/U. $\theta_{s}$ is the time-averaged flow separation angle measured from the upstream end of the cylinder (see Figure 7e). Backpressure coefficient is defined as follows,

$$
C_{p b}=\frac{p_{b}-p_{\infty}}{(1 / 2) \rho U^{2}}
$$

Here $p_{b}, p_{\infty}$, and $\rho$ are back pressure on the cylinder surface, free stream pressure and fluid density, respectively.

Time-averaged drag coefficient $\left(\mathrm{C}_{\mathrm{d}}\right.$ mean) predicted by CFD is smaller than the experimental value as seen in Table 2. Supporting this result, $\theta_{s}$ predicted is higher than the experimental. This implies that the wake width estimated by CFD is smaller. Even though the free stream TI in numerical simulation is rather small, the fully turbulent character of the k- $\omega$ SST model carries turbulence to the boundary layer, and possibly for this reason separation delays. CFD estimates $\mathrm{C}_{\mathrm{pb}}$ to be greater, confirming predictions in $\theta_{s}$ and $\mathrm{C}_{\mathrm{d} \text {-mean }}$. Rms of lift coefficient $\left(\mathrm{C}_{1-\mathrm{rms}}\right)$ estimated by CFD is much larger than the experimental value. In literature, employing 2D URANS simulations, fluctuating lift force was also 
computed larger than the experimental [17]. It is known that vortices behind the cylinder have 3D character and dislocate, this results in attenuation in vortices' strength [1]. The synchronous alternating vortices in 2D URANS simulations may be the reason for such a large fluctuating lift force. $\mathrm{fd}$ is calculated as twice of $\mathrm{fl}$ as in the literature [24][35]. St number determined by CFD is 0.267 and greater than the experimental value of 0.20 .

Table 2. Comparison of Computational Predictions and Experimental Results

\begin{tabular}{|c|c|c|c|c|}
\hline Parameter & Computational & $\begin{array}{l}\text { Experi } \\
\text { mental }\end{array}$ & $\begin{array}{l}\text { Reference } \\
\text { for Exp. } \\
\text { Data }\end{array}$ & Error \\
\hline $\mathrm{Cd}_{\text {d-mean }}$ & 1.056 & 1.181 & $\begin{array}{c}\text { Schlichting } \\
\text { and } \\
\text { Gersten } \\
{[13]}\end{array}$ & $-11 \%$ \\
\hline Cd-rms & 0.099 & $\mathrm{~N} / \mathrm{A}$ & & \\
\hline Cd-amp & 0.116 & $\mathrm{~N} / \mathrm{A}$ & & \\
\hline $\left.\mathrm{fd}_{\mathrm{d}} \mathrm{Hz}\right)$ & 0.293 & $\mathrm{~N} / \mathrm{A}$ & & \\
\hline Cl-rms & 0.909 & 0.511 & $\begin{array}{c}\text { Norberg } \\
{[36]}\end{array}$ & $78 \%$ \\
\hline $\mathrm{Cl-amp}$ & 1.264 & $\mathrm{~N} / \mathrm{A}$ & & \\
\hline $\mathrm{fl}(\mathrm{Hz})$ & 0.147 & 0.11 & $\begin{array}{c}\text { Roshko } \\
\text { [30] }\end{array}$ & $34 \%$ \\
\hline $\mathrm{St}^{* *}$ & 0.267 & 0.20 & $\begin{array}{c}\text { Roshko } \\
{[30]}\end{array}$ & $34 \%$ \\
\hline $\mathrm{Cpb}$ & -1.06 & -1.33 & $\begin{array}{c}\text { Williamson } \\
{[1]}\end{array}$ & $-20 \%$ \\
\hline$\theta_{s}$ & $\sim 110^{\circ}$ & $78^{\circ}$ & $\begin{array}{c}\text { Achenbach } \\
{[31]}\end{array}$ & $41 \%$ \\
\hline
\end{tabular}

**Strouhal number is based on frequency of lift force.

\subsection{Cross Flow over 8-Grooved Cylinder at $\operatorname{Re}=1,1 \times 10^{5}$}

An experimental work of El-Makdah and Oweis [18] is considered to test 2D URANS prediction capability of the flow field around a grooved cylinder. The experiments were carried out in an open wind tunnel. The grooved cylinder diameter is $69 \mathrm{~mm}$ as depicted in Figure 2a. The square test section has 45,5 cm side lengths. Therefore, in experiments, the aspect ratio of the grooved cylinder (L/D) and blockage ratio are 6.59 and $15 \%$, respectively.

$U$, air viscosity $(\mu)$ and air density $(\rho)$ are specified as $23.3 \mathrm{~m} / \mathrm{s}, 1.79 \times 10^{-5}$ Pa.s and $1.225 \mathrm{~kg} / \mathrm{m}^{3}$ in CFD solver. These parameters correspond to a Re number of $1.1 \times 10^{5}$ as in the experiment. The grooved cylinder transits the flow into the turbulence regime immediately after the first crest at $\theta=45^{\circ}$. For this reason, it is expected that laminarturbulence transition location is not critical for flow over the grooved cylinder. However 3D nature of both turbulence and vortex structures behind the cylinder still has an importance on fluctuating forces.

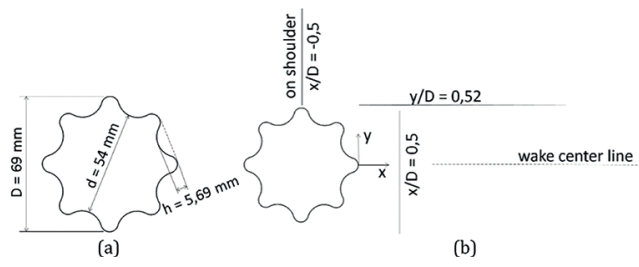

Figure 2. a) Dimensions of the 8-Grooved Cylinder (Reproduced from [18]) b) Origin, Axis Directions and, CFD Post-Processed Lines where Field Variables are Presented

Time-averaged streamwise velocity profiles are depicted in Figure 3. Backflow in the near wake cannot be captured by CFD as seen in Figure 3a. It was reported that RANS simulations fail to predict reversed mean flow in the cylinder wake [5]. 60\% of the free stream velocity recovers at a distance twice cylinder diameter both in the experiment and CFD. Wake recovers faster and wake width slightly wider in experimental results (Figure 3b). Mean shear in the wake is nearly the same for both cases. The maximum velocity in the shear layer is greater in experiments (Figure 3b). However, $U$ was measured $1,6 D$ upstream of the cylinder in experiments. With reference to the CFD solution, velocity is retarded 
from $U$ by $11 \%$ at this location. Therefore, streamwise velocity $(u)$ possibly is less than reported in experiments. On the other side, blockage ratios in the experiment and CFD are $15 \%$ and 1\%, respectively. Larger blockage ratio in the experiment may be the other consideration of higher velocity.

Time-averaged TI profiles on the vertical line (at $x / D=0.5$ ) are shown in Figure 4a. TI predicted by CFD is much smaller than the experimental results. Free stream TI in the wind tunnel tests is not known. On the other hand, after comparison RANS simulations with LES and PIV experiments, Stetson [5] reported that Reynolds averaging in RANS modelling smooths out instantaneous peaks in turbulence fluctuations. This results in lower predicted fluctuating loads [5]. Magnitude and change in spanwise vorticity $\left(\omega_{z}\right)$ along upper and lower shear layers agree well with experiments as seen in Figure 4b. Vorticity magnitudes close to the cylinder is higher in CFD. Greater vorticity strength in the neighborhood of the cylinder may result in increased predicted fluctuating lift loads in CFD [19].

\section{3. Influence of Number of Grooves}

\subsubsection{Time Statistics}

To investigate the effects of the number of grooves on hydrodynamic forces and flow field, 8-, 10- and, 12-grooved cylinders are considered. The 8-grooved cylinder in the previous section is scaled to be 0,5 $\mathrm{m}$ in diameter. Three cylinders with their dimensions are given in Table 3. In CFD, seawater cross flow over the three cylinders are simulated at $\operatorname{Re}=10^{5} . U, \mu$ and $\rho$ are $0.275 \mathrm{~m} / \mathrm{s}, 1.41 \times 10^{-3}$ Pa.s and $1.027 \mathrm{~kg} / \mathrm{m}^{3}$, respectively.

Parameters of fluctuating drag and lift forces are provided in Table 3. After discarding the initial transient force data, the periodically repeated parts of force signals in Figures $5 \mathrm{a}$, 5b, 5c and Figures 6a, 6b, 6c are utilized in Fast Fourier Transform (FFT) analyses. FFT function embedded in Matlab is employed to construct the frequency spectrum of timevarying force data. Kreyszig [40] summarized the mathematical theory behind FFT. The frequency spectrum of fluctuating drag and lift forces are presented in Figures 5d, 5e, $5 f$ and Figures 6d, 6e, 6f, respectively. As the number of grooves increases, a slight decrease in $\mathrm{C}_{\mathrm{d} \text {-mean }}$ is observed. $\mathrm{C}_{\mathrm{d} \text {-rms }}$ of the 12-grooved cylinder is minimum and $29 \%$ smaller than that of 8-grooved cylinder. In the same way, the amplitude of drag coefficient $\left(\mathrm{C}_{\mathrm{d} \text {-amp }}\right)$ of the 12-grooved cylinder is less than the 8-grooved cylinder by $35.5 \%$. However, $\mathrm{C}_{\mathrm{d} \text {-rms }}$ and $\mathrm{C}_{\mathrm{d} \text {-amp }}$ of 10 -grooved cylinder increased by $44 \%$ and $30 \%$, respectively. Comparing with the dominant frequency of drag force $\left(\mathrm{f}_{\mathrm{d}}\right)$ of the 8-grooved, $\mathrm{f}_{\mathrm{d}}$ of the 10-grooved decreases by $69 \%$, and $f_{d}$ of the 12 -grooved remains the same.

While the dominant frequency of lift coefficient $\left(\mathrm{f}_{1}\right)$ of 8-, 10, -12-grooved cylinders are almost the same, $\mathrm{C}_{1-\mathrm{rms}}$ values for the 10 -grooved and the 12-grooved increase by $12 \%$ and $24 \%$, respectively. Lift forces on 8 and 12-grooved cylinders are sinusoidally changing, but that of the 10-grooved has two strong peaks in amplitude spectrum and therefore it is the sum of two sinusoidal force signals. St number of the three cylinders is changing between 22 and 25 (Table 3).

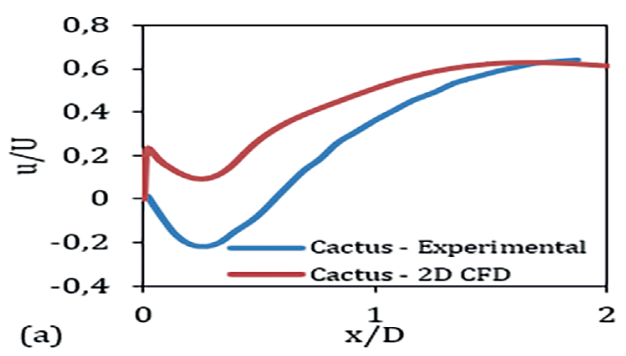

Figure 3. Comparison of Time-Averaged Streamwise Velocity a) along the Wake Center Line 


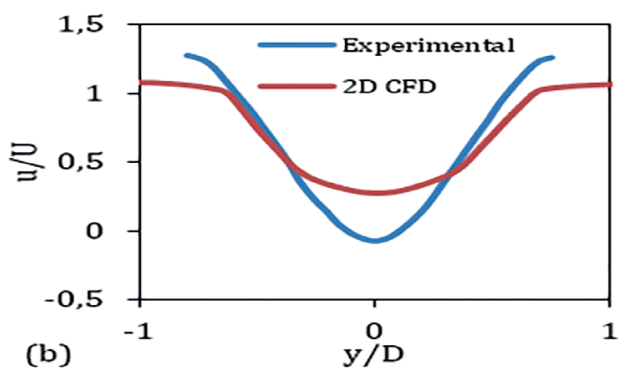

Figure 3. b) along Vertical Line at $x / D=0.5$. Experimental Data is from [18].
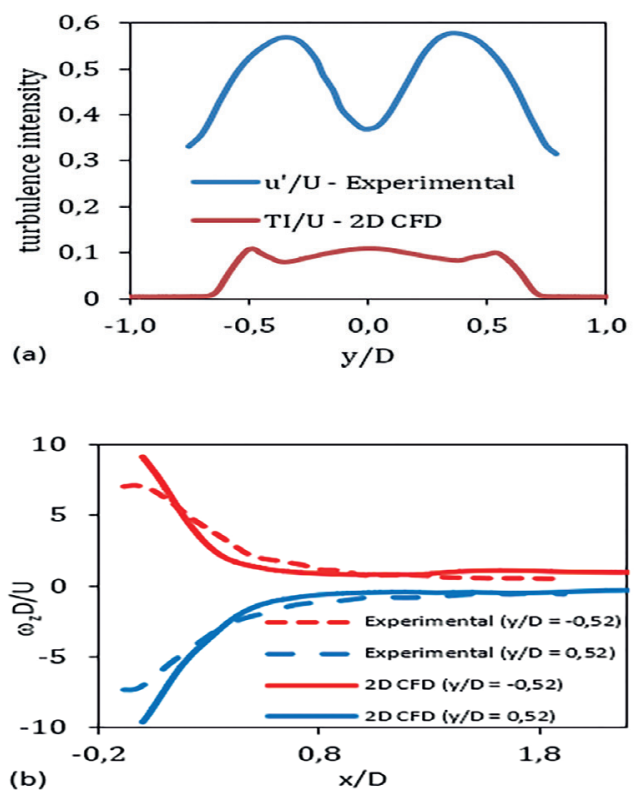

Figure 4. Comparison of Flow Field Variables. a) Time-Averaged TI along Vertical Line at $x / D=0.5$. Time Average of $\mathbf{T I}=\sqrt{\mathbf{0 , 5}\left(\boldsymbol{u}^{\prime 2}+\boldsymbol{v}^{\prime 2}\right)}$ is Plotted. $\left.\boldsymbol{b}\right)$ Time-Averaged $\omega_{z}$ along Horizontal Line at $y / D=$ \pm 0.52 . Experimental Data is from [18].

\subsubsection{Time-Averaged Variables}

Time-averaged line plots based on 2D URANS simulations are shown in Figure 7. To generate time-averaged line plots, 20 snapshots per shortest time period of fluctuating forces are captured (this is the sampling rate) and a 5-10 repeated part of force signal with the longest time period is utilized. Streamwise velocities of 10-, and 12-grooved cylinders at the wake centerline reach slightly larger values at $x / D=2$ (Figure $7 a$ ). This result implies reduced drag force with reference to 8-grooved cylinder [18]. Mean spanwise vorticity near the cylinders decreases as the number of grooves increases as seen in Figure 7b. As the flow moved away from the cylinders, the vorticity magnitudes converge to each other and, vorticity receives slightly greater value for the 10-grooved. Shear on grooved cylinders is expected to be less than the smooth cylinder because effective friction surface of grooved cylinders is smaller. This leads to a decrease in vortex strength [18]-[21]. Mean shear on grooved cylinder shoulders (at $x / D=-0.52$ in Figure $2 \mathrm{~b}$ ) is depicted in Figure $7 \mathrm{c}$. Since the cavity of the 10-grooved is located on the shoulder, its velocity gradient (shear) is less than the others. Mean TKE on the cylinder shoulder increases by the number of grooves as depicted in Figure 7d. Increased momentum in boundary layers due to turbulence helps the flow to overcome adverse pressure gradient on curved surfaces and delay separation [13] [14][37]. This is the main reason for reduced $\mathrm{C}_{\mathrm{d} \text {-mean }}$ by an increase in the number of grooves. Time-averaged pressure coefficients $\left(\mathrm{C}_{\mathrm{p}}\right)$ around the cylinders are depicted in Figure 7e. Lack of perfect symmetry in pressure distribution is possibly related to the sampling rate of time-varying data. $\theta_{s}$ increases with the number of grooves. This result again confirms the reduced drag by the number of grooves. Yamagishi and Oki [37] showed that more grooves on triangular grooved cylinders reduced critical Re number where $C_{d}$ abruptly drops due change in boundary layer turbulence. As seen in Table $3, \mathrm{C}_{\mathrm{pb}}$ increases by the number of grooves verifying smaller drag. Due to the absence of spanwise flow in cylinders' wake of a 2D CFD computation domain, $\mathrm{Cpb}$ values are probably smaller than the values that can be obtained by $3 \mathrm{D}$ computations [1]. 
Table 3. Geometry Data, Fluctuating Force Coefficients, Frequency of Forces, and Time-Averaged $C_{p b}$ and $\theta_{s}$ for Cross Flow of Seawater over Grooved Cylinders at Re $=10^{5}$

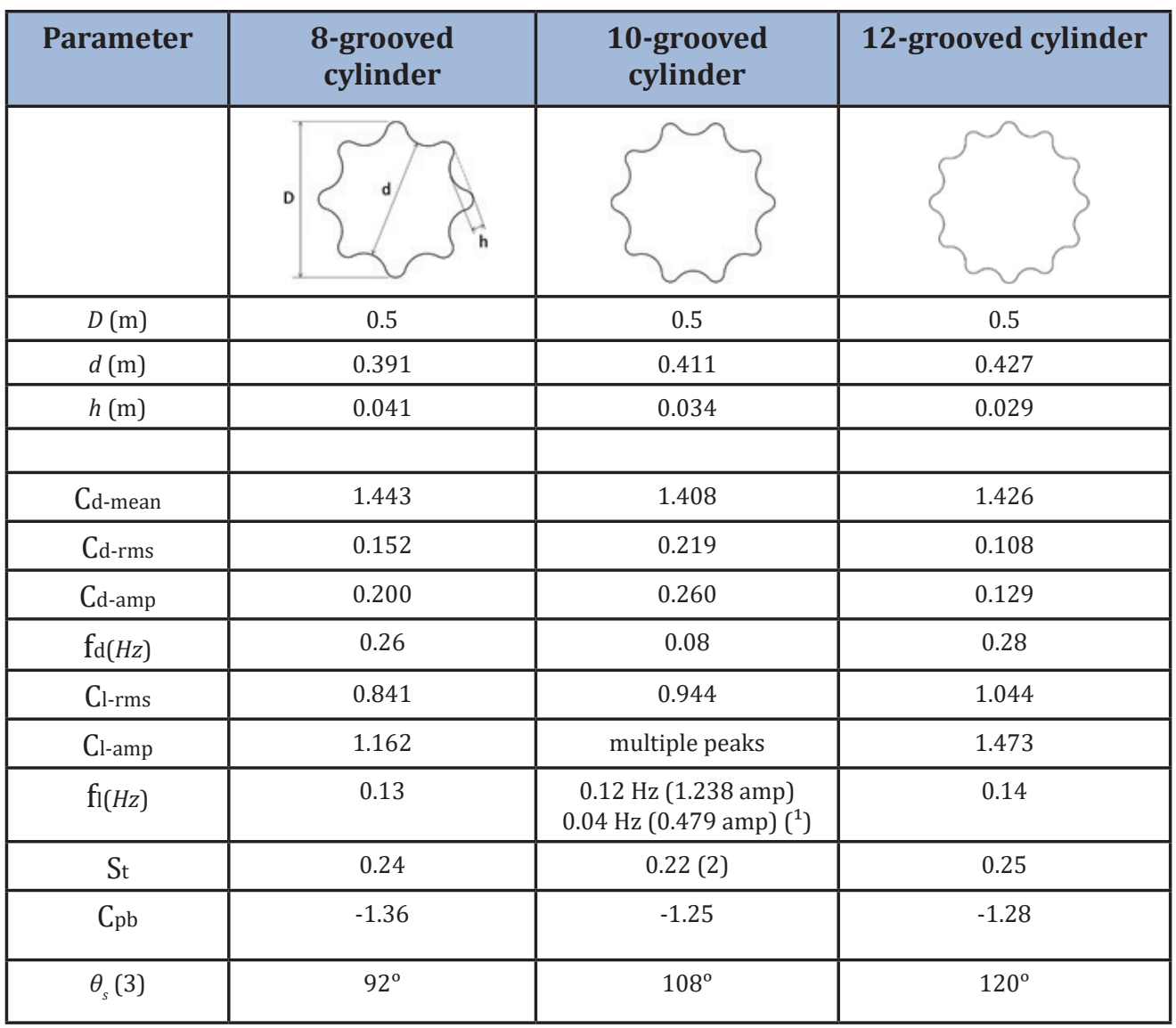

${ }^{1}$ Multiple peaks exist in amplitude spectrum of lift coefficient $\left(\mathrm{C}_{1}\right)$.

${ }^{2}$ St number is specified based on the dominant frequency of $\mathrm{C}_{1}$ with maximum amplitude.

${ }^{3} \theta_{s}$ is determined based on time-averaged $\mathrm{C}_{\mathrm{p}}$ distribution around the cylinders. $\theta_{s}$, as an effective separation angle, is specified when $\mathrm{C}_{\mathrm{p}}$ starts to level off. There is no increase in $\mathrm{C}_{\mathrm{p}}$ after this specified angle (see Figure 7e).

\subsubsection{Instantaneous Flow Field}

Instantaneous contours and velocity vectors for maximum lift force are shown in Figure 8. The high streamwise velocity, high vorticity [18] and separated flow are responsible for low pressure on upper surfaces of cylinders resulting lift force in $+y$ direction. High-pressure stagnation zone at the front end of the cylinders is largest for the 8-grooved.
Vortices wrap around 12-grooved cylinder more closely. As a result, the flow separates at a larger angular position compared to the 8-, and 10-grooved cylinders. When vortices form farther than the cylinder, fluctuating side forces lessen [12],[19]. With reference to the conservation principle of momentum, flow stream of fluids exert force on the solid body in the opposite direction of flow [38]. Downward $\mathrm{v}$-velocity (Blue colours of $\mathrm{v}$-velocity contours 
indicate downward velocity) occupies larger region for the 12-grooved as seen in contours of v-velocity in Figure 8. In the meantime, if the contours of $\mathrm{v}$-velocity and velocity vector plot are examined, downward flow stream (downwash) for the 12-grooved is more closely compared to the other cylinders. These two points explain the greater fluctuating lift force on the 12-grooved cylinder regarding 2D URANS results.

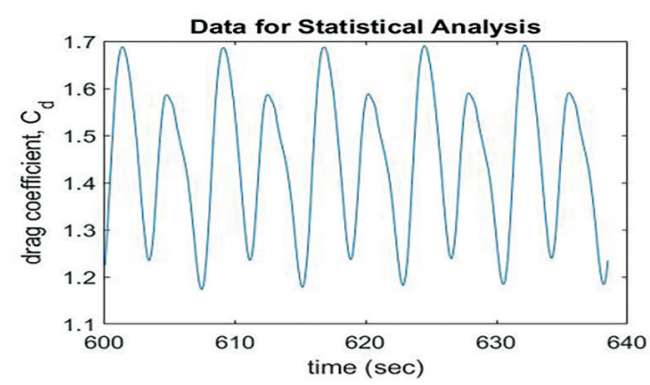

a)

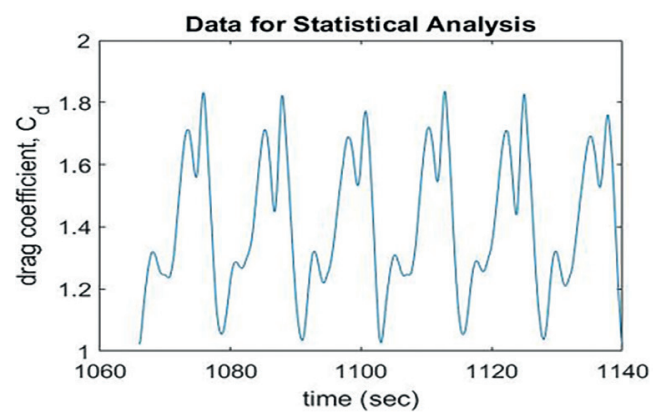

b)

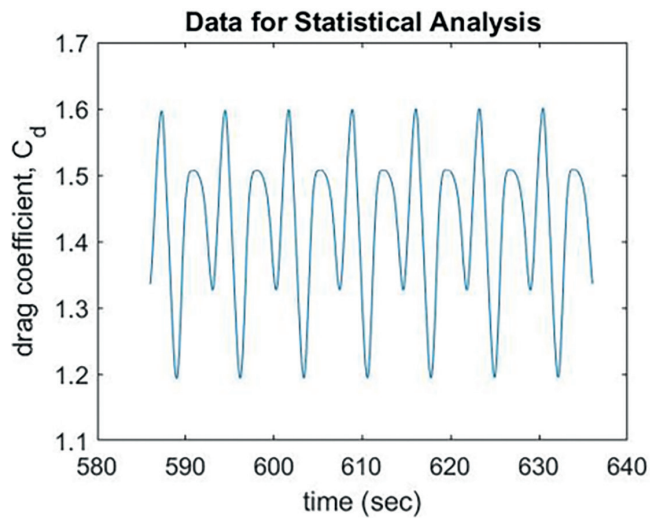

c)

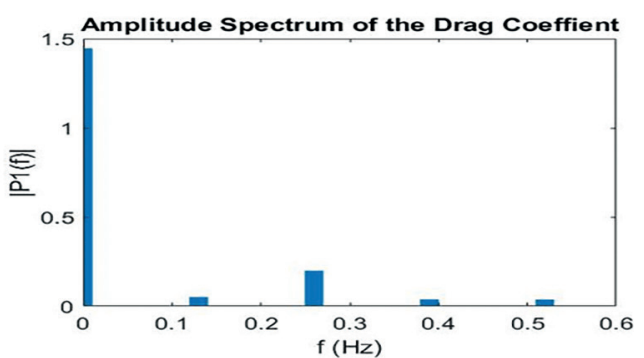

d)

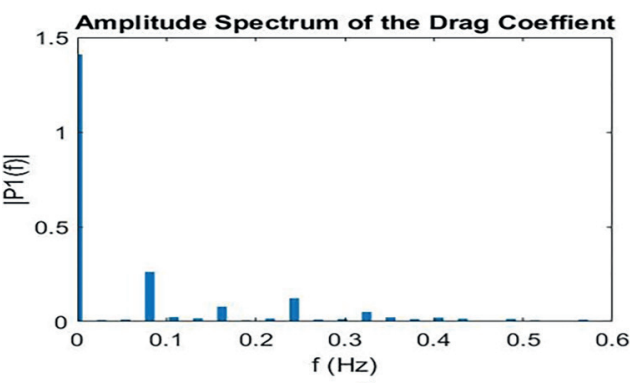

e)

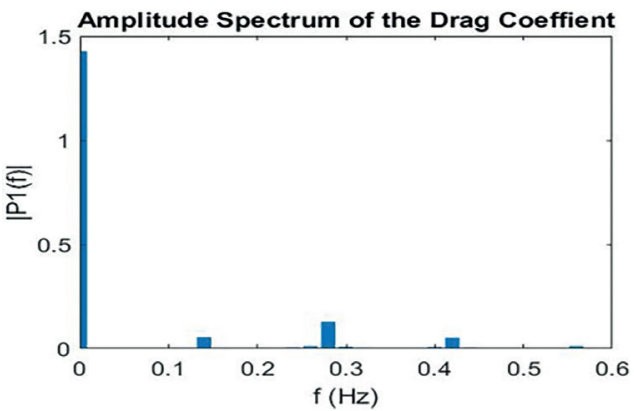

f)

Figure 5. Time Series $(\boldsymbol{a}, \boldsymbol{b}, \boldsymbol{c})$ and Amplitude Spectrum $(\boldsymbol{d}, \boldsymbol{e}, \boldsymbol{f})$ of Drag Coefficient for 8-Grooved Cylinder $(\boldsymbol{a}, \boldsymbol{d}), 10$-Grooved Cylinder $(\boldsymbol{b}, \boldsymbol{e})$, and 12-Grooved Cylinder $(\boldsymbol{c}, \boldsymbol{f})$

Turbulence intensity contours show that the boundary layer gets into turbulence just after the groove of the stagnation region at the front end. The energized boundary layer can withstand pressure forces and flow separation is delayed.

For such a high Re number flow, the 
hydrodynamic lift forces arise solely due to asymmetric instantaneous pressure distribution around the cylinders [1]. Friction force contribution to hydrodynamic forces for high Re is negligibly small [11][20]. In Figure $7 \mathrm{e}$, as expected, time-averaged $\mathrm{C}_{\mathrm{p}}$ around the cylinders is almost symmetrical. When the snapshots of the $\mathrm{C}_{\mathrm{p}}$ distribution around the cylinders are considered, they should likely be asymmetrical due to alternating shedding vortices on the downstream side of the cylinders. Resulting pressure distributions of the grooved cylinders for maximum lift force are depicted in Figure 9. The area between $C_{p}$ curves of upper and lower surfaces is proportional to lift force [39]. Confirming the reported fluctuating lift force $\left(\mathrm{C}_{1-\text {-rms }}\right)$ data in Table 3, area for the 12-grooved cylinder is largest and that for the 8-grooved is smallest. From Figure 9, the instantaneous separation angles of upper and lower surfaces can be determined detecting start point of leveloff in $C_{p}$ distribution. In this regard, after cross-check with contours of $u$-velocity and vorticity in Figure 8, instantaneous separation angles of upper and lower surfaces of 8-, 10- and 12-grooved cylinders are specified as $\left(90^{\circ}, 90^{\circ}\right),\left(108^{\circ}, 72^{\circ}\right)$ and $\left(120^{\circ}, 90^{\circ}\right)$, respectively.

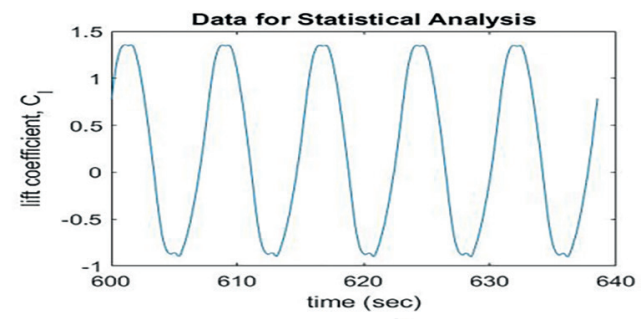

a)

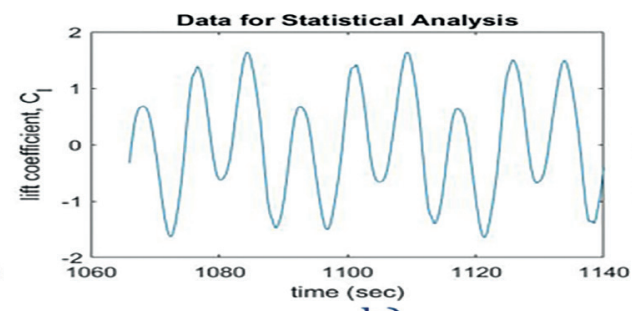

b)

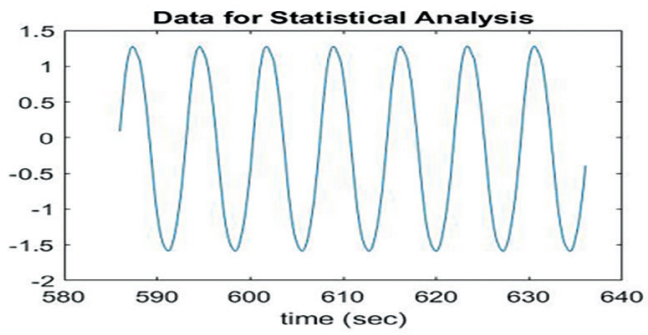

c)

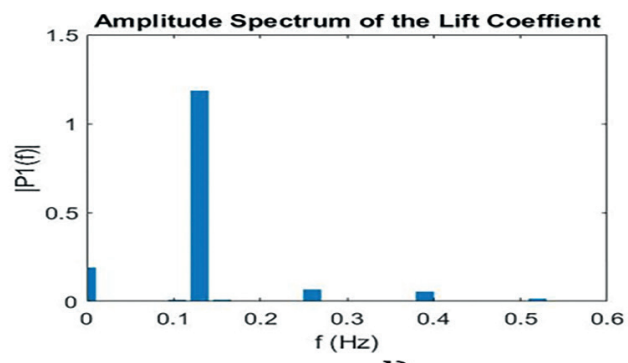

d)

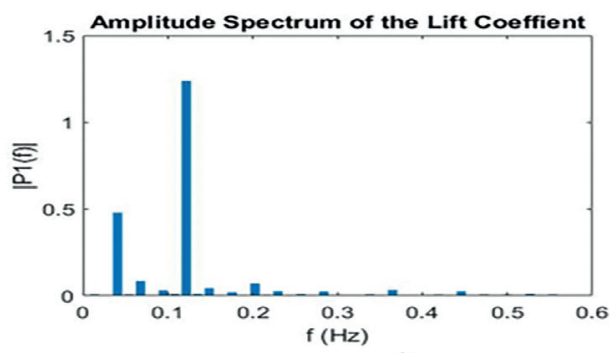

e)

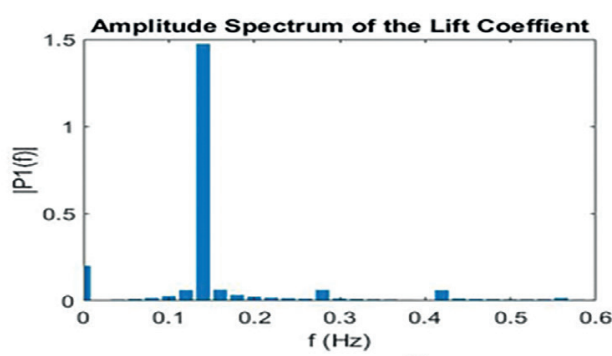

f)

Figure 6. Time Series $(\boldsymbol{a}, \boldsymbol{b}, \boldsymbol{c})$ and Amplitude Spectrum $(\boldsymbol{d}, \boldsymbol{e}, \boldsymbol{f})$ of Lift Coefficient for 8-Grooved Cylinder (a,d), 10-Grooved Cylinder $(\boldsymbol{b}, \boldsymbol{e})$, and 12-Grooved Cylinder $(\boldsymbol{c}, \boldsymbol{f})$ 

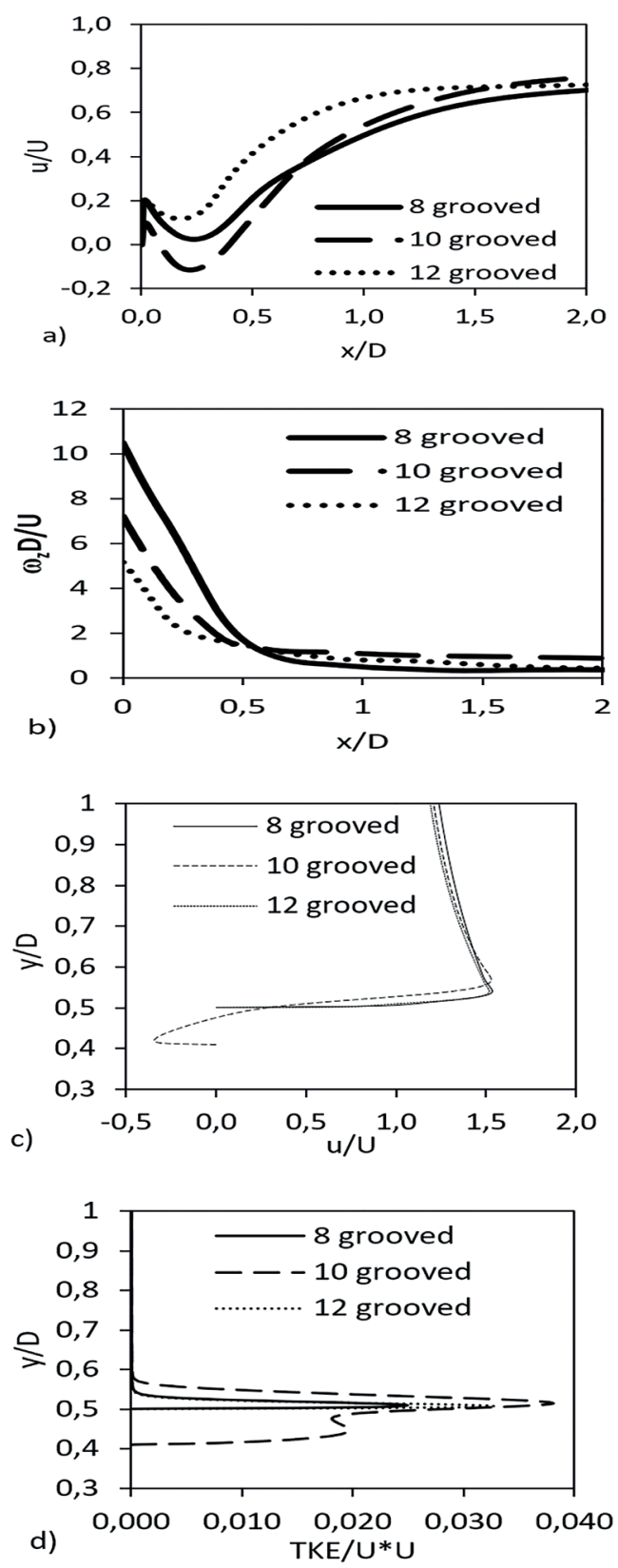

Figure 7. Time-Averaged Line Plots based on $2 D$ URANS Simulations a) Streamwise Velocity on the Wake Center Line b) $\omega z$ along Horizontal Line at $y / D=0.52$ c) Velocity along Vertical Line on the Shoulder $(x / D=-0.5)$ d) TKE along Vertical Line on the Shoulder $(x / D=-0.5)$

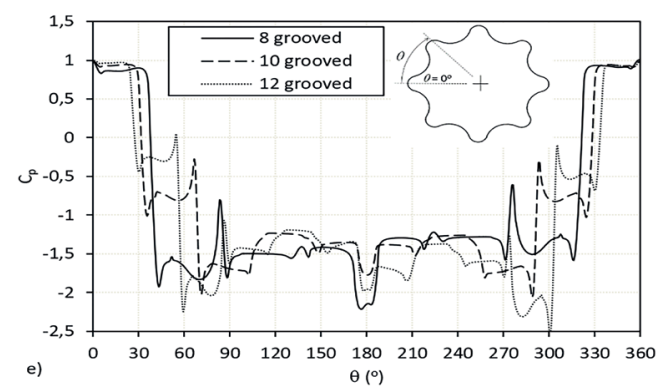

Figure 7. (continued) Time-Averaged Line Plots based on $2 D$ URANS Simulations e) $\mathrm{C}_{\mathrm{p}}$ around the Cylinders

\section{Conclusion}

In this study, the influence of the number of rounded grooves on cylinders is investigated employing 2D URANS computations. The first unsteady CFD analysis is performed on a smooth cylinder at $\operatorname{Re}=10^{5}$. While separation angle $\left(\theta_{s}\right)$ is predicted larger than the experimental, consistently, $\mathrm{C}_{\mathrm{d} \text {-mean }}$ and base suction coefficient $\left(-\mathrm{C}_{\mathrm{pb}}\right)$ are estimated smaller compared to the experimental results. Rms of $\mathrm{C}_{1}$ is calculated larger because the flow is solved with 2D CFD analysis, which cannot consider spanwise flow and vortex dislocations in the wake field of the smooth cylinder.

The second CFD analysis is carried out on an 8-grooved cylinder at $\operatorname{Re}=1.1 \times 10^{5}$. Although the backflowin the near wakecannot be predicted, recovered velocity magnitude is in close agreement with experiment. Mean shear and mean vorticity profiles in the shear layer computed by CFD show a good match with the literature experimental results.

The third CFD work is conducted on 8-,10, and 12-grooved cylinders. While $\mathrm{C}_{\mathrm{d} \text {-mean }}$ of the 12-grooved cylinder is slightly smaller compared to the 8-grooved, its fluctuating drag $\left(\mathrm{rms}\right.$ of $\mathrm{C}_{\mathrm{d}}$ ) is 29\% smaller. Although rms of $\mathrm{C}_{\mathrm{d}}$ of the 10 -grooved increases by $44 \%$ compared to the 8-grooved, its dominant frequency of $\mathrm{C}_{\mathrm{d}}$ decreases considerably by $69 \%$. $\theta_{s}$ has been significantly improved from $92^{\circ}$ of the 8 -grooved to $120^{\circ}$ of 
the 12-grooved. Turbulence intensity contours reveal the immediate transition to turbulence after the first grooves. In this way, TKE increases by the number of grooves and this is followed by a rise in $\theta_{s^{\prime}}$ On the other hand, $r m s$ of $C_{1}$ of the 12-grooved increases by $24 \%$ compared with the 8-grooved. Closer vortex rollup for the 12-grooved results in stronger downwash, in turn, more lift force arises with reference to conservation of momentum. Frequency of lift remains nearly the same for the grooved cylinders.

In summary, experimental time statistics of fluctuating forces are partially reproduced in 2D CFD for a smooth cylinder. Time-averaged vorticity and velocity profiles on the shear layer of the 8-grooved cylinder are fairly well predicted by 2D CFD comparing with experimental results. However, backflow in the immediate wake of the 8-grooved cannot be predicted.
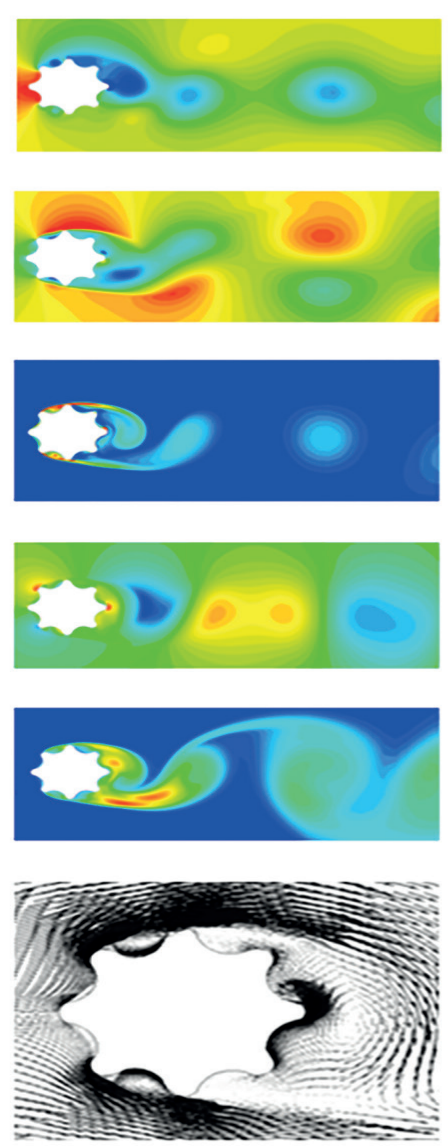

a)

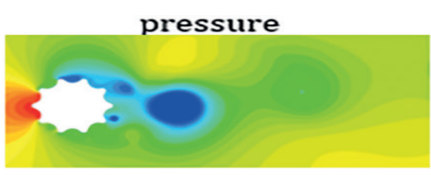

$u$-velocity

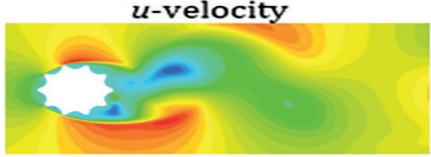

vorticity magnitude

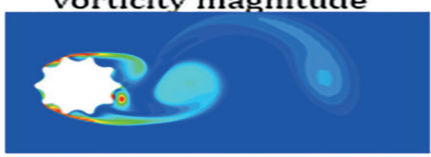

v-velocity

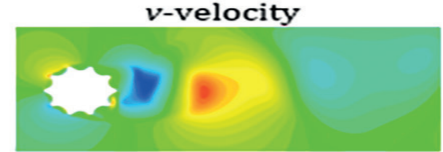

turbulence intensity

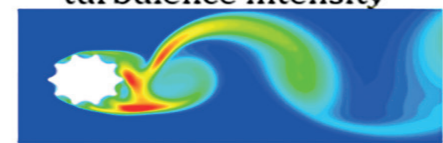

velocity vectors

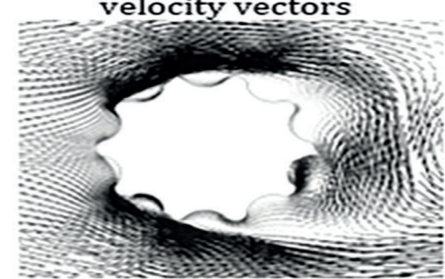

b)
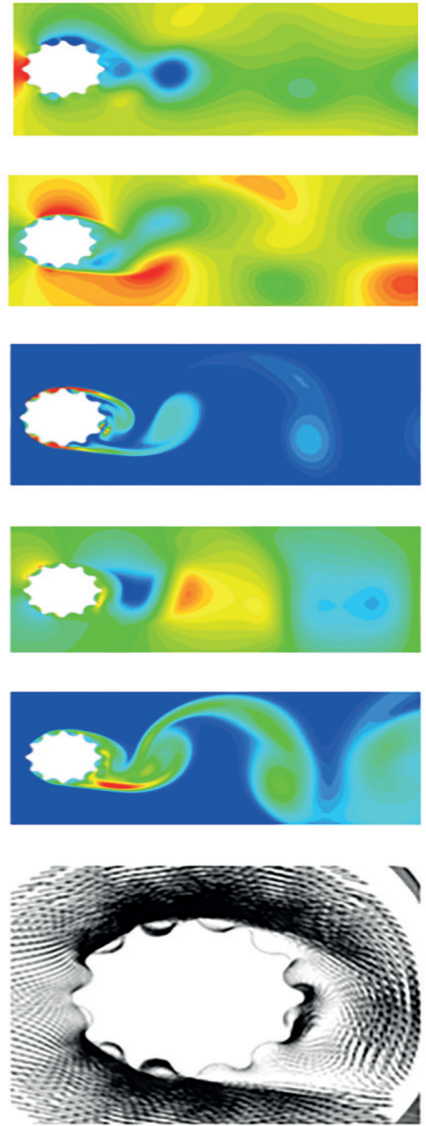

c)

lowest

highest

Figure 8. Instantaneous Contours and Velocity Vector for Maximum Lift Force a) 8-Grooved Cylinder $\left(\mathrm{C}_{1}=\right.$ 1.34) b) 10-Grooved Cylinder $\left(\mathrm{C}_{1}=1.46\right)$ c) 12-Grooved Cylinder $\left(\mathrm{C}_{1}=1.59\right)$ 


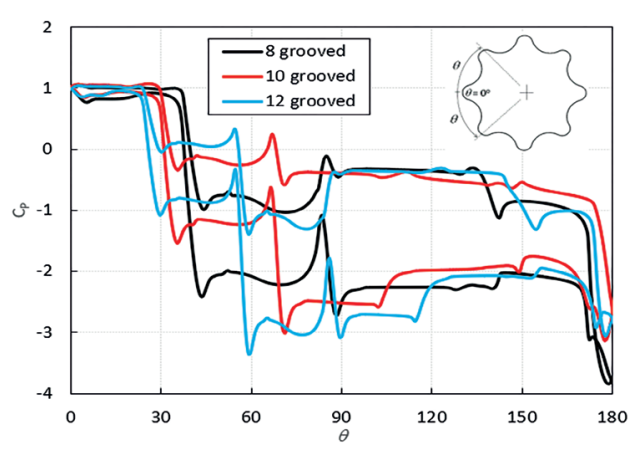

Figure 9. Instantaneous Pressure Coefficient around the Cylinder for Maximum Lift Force

TI level computed in 2D CFD is well below that of experiments possibly due to Reynolds averaging in RANS modelling. The ability of grooved cylinders to reduce the fluctuating lift force observed in experimental literature is not seen in 2D CFD analysis. Fluctuating lift force is overestimated due to inability of 2D CFD to take into account vortex dislocations. It is shown and discussed that the increased number of rounded grooves has the potential to improve the hydrodynamic drag characteristics of a cylindrical structure. Although 2D CFD results are consistent in itself, it is believed and partially seen that 2D CFD approach are deficient predicting highly complicated flow field around smooth and grooved cylinders. Therefore 3D CFD approach, which will be more proper choice of research tool, is essential to extend the research and investigate the benefits of grooves.

\section{Acknowledgement}

The author would like to thank Eng. Şafak Doğru (M.Sc.) from Numesys Inc. for his valuable suggestions on the mesh structure in Ansys Meshing.

\section{References}

[1] Williamson, C. (1996). Vortex Dynamics in the Cylinder Wake. Annu. Rev. Fluid Mech., 28 (1), 477-539. https://doi.org/10.1146/annurev. fluid.28.1.477.

[2] Zhou, T., Razali, S. F. M. \& Cheng, L. (2010). Investigation on Suppression of Vortex-Induced Vibration Using Helical Strakes. 29th International Conference on Ocean, Offshore and Arctic Engineering. Shanghai: ASME. https://doi.org/10.1115/OMAE201020984

[3] Quen, L. K., Abu, A., Kato, N., Muhamad, P., Sahekhaini, A., \& Abdullah, H. (2014). Investigation on the Effectiveness of Helical Strakes in Suppressing VIV of Flexible Riser. Appl. Ocean Res, 44, 82-91. http://dx.doi.org/10.1016/j. apor.2013.11.006.

[4] Allen, D. W., \& Allen, C. W. (2008). Fairings vs. Helical Strakes for Suppression of Vortex-Induced Vibration: Installation, Maintenance, and Economic Considerations. Offshore Technology Conference. Houston. https://doi.org/10.4043/19374-MS.

[5] Stetson, P. B., \& Kinnas, S. A. (2013). PIV Measurements of Flow-Field Downstream of a Cylinder in Uniform Flow and Comparisons with CFD (MSC Thesis). The University of Texas at Austin.

[6] Huang, S., \& Sworn, A. (2013). Hydrodynamic Coefficients of Two Fixed Circular Cylinders Fitted with Helical Strakes at Various Staggered and Tandem Arrangements. Appl. Ocean Res, 43, 21-26. http://dx.doi. org/10.1016/j.apor.2013.06.001.

[7] Owen, J. C., Bearman, P. W., \& Szewczyk, A. A. (2001). Passive Control of VIV With Drag Reduction. J. Fluids Struct., 15 (3-4), 597-605. https://doi. org/10.1006/jfls.2000.0358.

[8] Allen, D. W., \& Henning, D. (2008). 
Comparisons of Various Fairing Geometries for Vortex Suppression at High Reynolds Numbers. Offshore Technology Conference. Houston. https://doi.org/10.4043/19377ms.

[9] Zhang, Daichin, W., \& Lee, S. J.(2005). PIV Measurements of the Near-wake behind a Sinusoidal Cylinder. Exp. Fluids, 38 (6), 824-832. https://doi. org/10.1007/s00348-005-0981-9.

[10] Nakamura, H., \& Igarashi, T. (2007). Reductions in Drag and Fluctuating Forces for a Circular Cylinder by Attaching Cylindrical Rings. J. Fluid Sci. Technol, 2 (1), 12-22. https:// doi.org/10.1299/jfst.2.12.

[11] Achenbach, E. (1971). Influence of Surface Roughness on the Cross-flow around a Circular Cylinder. J. Fluid Mech, 46 (2), 321-335. https://doi.org/10.1017/ s0022112071000569.

[12] Lam, K., Wang, F., \& So, R. M. (2004). Three-dimensional Nature of Vortices in the Near Wake of a Wavy Cylinder. J. Fluids Struct, 19 (6), 815833. https://doi.org/10.1016/j. jfluidstructs.2004.04.004.

[13] Schlichting, H., \& Gersten, K. (2017). Boundary-Layer Theory (9th ed). Berlin: Springer.

[14] Bushnell, D. (1991). Drag Reduction In Nature. Annu. Rev. Fluid Mech, 23 (1), 65-79. https://doi. org/10.1146/annurev.fluid.23.1.65.

[15] Lim, H.-C., \& Lee, S.-J. (2002). Flow Control of Circular Cylinders with Longitudinal Grooved Surfaces. AIAA J, 40 (10), 2027-2036. https://doi.org/10.2514/2.1535.

[16] Ko, N. W. M., Leung, Y. C., \& Chen, J. J. J. (1987). Flow Past V-groove Circular Cylinders. AIAA $J, 25$ (6), 806-811. https://doi. org/10.2514/3.9704.

[17] Talley, S., Iaccarino, G., Mungal,
G., \& Mansour, N. (2001). An Experimental and Computational Investigation of Flow Past Cacti. Center for Turbulence Research, Mech. Engg. Dept (Annual Research Briefs, pp. 51-63). Stanford University. Available from https:// web.stanford.edu/group / ctr / ResBriefs01/talley.pdf.

[18] El-Makdah, A. M., \& Oweis, G. F. (2013). The Flow Past a Cactusinspired Grooved Cylinder. Exp. Fluids, 54 (2). https://doi. org/10.1007/s00348-013-1464-z.

[19] Liu, Y. Z., Shi, L. L., \& Yu, J. (2011). TR-PIV Measurement of the Wake behind a Grooved Cylinder at Low Reynolds Number. J. Fluids Struct, 27 (3), 394-407. https://doi.org/10.1016/j. jfluidstructs.2010.11.013.

[20] Morgenthal, G., \& Walther, J. H. (2007). An Immersed Interface Method for The VortexIn-Cell Algorithm. Comput. Struct, 85 (11-14), 712-726. https://doi.org/10.1016/j. compstruc.2007.01.020.

[21] Babu, P., \& Mahesh, K. (2008). Aerodynamic Loads on Cactusshaped Cylinders at Low Reynolds Numbers. Phys. Fluids, 20 (3), 1-9. https://doi. org/10.1063/1.2887982.

[22] Abboud, J. E., Karaki, W. S., \& Oweis, G. F. (2011). Particle Image Velocimetry Measurements in the Wake of a Cactus-Shaped Cylinder. J. FluidsEng,133 (9), 094502.https:// doi.org/10.1115/1.4004824.

[23] Talley, B. S., \& Mungal, G. (2002). Flow around Cactus-shaped Cylinders. Center for Turbulence Research, Mech. Engg. Dept (Annual Research Briefs, pp. 363-376). Stanford University. Available from https://web.stanford. edu/group/ctr/ResBriefs02/talley.pdf 
[24] Trim, A. D., Braaten, H., Lie, H., \& Tognarelli, M. A. (2005). Experimental Investigation of Vortex-induced Vibration of Long Marine Risers. J. Fluids Struct, 21 (3), 335-361. SPEC. ISS. https://doi.org/10.1016/j. jfluidstructs.2005.07.014.

[25] Ansys Fluent. (2019). R2, from http:// www.ansys.com.

[26] Menter, F. R. (1992). Improved Twoequation K-omega Turbulence Models for Aerodynamic Flows. NASA Tech. Memo, 103978, 1-31. California. Available from https://ntrs.nasa. gov/archive/nasa/casi.ntrs.nasa. gov/19930013620.pdf

[27] Ansys Fluent Theory Guide 16.1.

[28] Aranake, A. C., Lakshminarayan, V. K., \& Duraisamy, K. (2015). Computational Analysis of Shrouded wind Turbine Configurations using a 3-dimensional RANS Solver. Renew. Energy, 75, 818-832. https://doi.org/10.1016/j. renene.2014.10.049.

[29] Sumer, B. M., \& Fredsøe, J. (2006). Hydrodynamics Around Cylindrical Structures (revised ed), 26. New Jersey: World Scientific.

[30] Roshko, A. (1961). Experiments on the Flow Past a Circular Cylinder at Very High Reynolds Number. J. Fluid Mech, 10 (3), pp. 345-356. https://doi. org/10.1017/S0022112061000950.

[31] Achenbach, E. (1968). Distribution of Local Pressure and Skin Friction around a Circular Cylinder in CrossFlow up to $\mathrm{Re}=5 \times 10$ 6. J. Fluid Mech, 34 (4), 625-639. https://doi. org/10.1017/S0022112068002120.

[32] Bearman, P. W. (1969). On Vortex Shedding from a Circular Cylinder in the Critical Reynolds Number Rágime. J. Fluid Mech, 37 (3), 577585. https://doi.org/10.1017/ S0022112069000735.

[33] Dong, S., \& Karniadakis, G. E. (2005). DNS of Flow Past a Stationary and
Oscillating Cylinder at $\mathrm{Re}=10000$. J. Fluids Struct, 20 (4), 519-531. SPEC. ISS. https://doi.org/10.1016/j. jfluidstructs.2005.02.004.

[34] Bearman, P. W. (2009). Understanding and Predicting Vortex-induced Vibrations. J. Fluid Mech, 634, 1-4.https://doi.org/10.1017/ S0022112009990851.

[35] Ranjith, E. R., Sunil, A. S. \& Pauly, L. (2016). Analysis of Flow over a Circular Cylinder Fitted with Helical Strakes. Procedia Technol, 24, 452460. https://doi.org/10.1016/j. protcy.2016.05.062.

[36] Norberg, C. (2001). Flow Around a Circular Cylinder: Aspects of Fluctuating Lift. J. Fluids Struct, 15 (3-4), 459-469. https://doi. org/10.1006/jfls.2000.0367.

[37] Yamagishi, Y. \& Oki, M. (2005). Effect of the Number of Grooves on Flow Characteristics around a Circular Cylinder with Triangular Grooves. J. Vis, 8 (1), 57-64. https://doi. org/10.1007/BF03181603.

[38] Çengel, Y. A., \& Cimbala, J. M. (2013). Fluid Mechanics: Fundamentals and Applications, 3rd ed. New York: McGraw-Hill.

[39] Somers, D. M. (1997). Design and Experimental Results for the S809 Airfoil. Natl. Renew. Energy Lab. NREL, NREL/SR-44, Golden, Colorado. Available from https://www.nrel.gov/ docs/legosti/old/6918.pdf

[40] Kreyszig, E. (2011). Advanced Engineering Mathematics (10th ed.) New Jersey: John Wiley \& Sons. 